\title{
ANALYTICAL METHODS FOR A SINGULAR PERTURBATION PROBLEM IN A SECTOR*
}

\author{
N. M. TEMME $\dagger$
}

\begin{abstract}
From the exact solution of an elliptic boundary value problem in a sector, asymptotic approximations with respect to a small parameter are derived. The asymptotic expansion is uniformly valid in the boundary layers. Also the phenomena for the case of almost characteristic boundaries are discussed.
\end{abstract}

1. Introduction. In [4], the author considered a singular perturbation problem for an elliptic equation in a quarter-plane. The exact solution of the equation was represented as a contour integral and from this representation the asymptotic solution was derived by using saddle point methods.

In this paper we consider the same equation

$$
\varepsilon \Delta \Phi_{\varepsilon}(x, y)-\frac{\partial}{\partial y} \Phi_{\varepsilon}(x, y)=0
$$

the domain of definition now being an arbitrary sector shaped domain in the $x, y$-plane

$$
A=\{r, \phi \mid r \geqq 0,0 \leqq \phi \leqq \alpha\} .
$$

In (1.1) $\varepsilon$ is a small positive parameter and $\Delta$ is Laplace's operator; in (1.2) $r$ and $\phi$ are polar coordinates, where

$$
x=r \cos \phi, \quad y=r \sin \phi
$$

and

$$
0<\alpha \leqq 2 \pi
$$

The case $\alpha=\frac{1}{2} \pi$ (the quarter-plane) is discussed in [4].

Along the boundary of the sector $A$, the function $\Phi_{\varepsilon}$ is subjected to the following boundary conditions :

$$
\Phi_{\varepsilon}(x, 0)=0, \quad \Phi_{\varepsilon}(x, y)=1 \quad \text { if } \phi=\alpha .
$$

In order to investigate the asymptotic behavior for small values of $\varepsilon$, the exact solution of (1.1) is determined from which the asymptotic approximations are derived. Also the various types of boundary layers are discussed, for instance the "free" (i.e., internal) boundary layer in the case of an obtuse angle $\alpha$. Finally, the case of an almost right angle will be considered.

2. The solution of the boundary value problem. We shall first remove the first order derivative in equation (1.1) by substituting

$$
\Phi_{\varepsilon}(x, y)=1-e^{\omega y} F(x, y), \quad \omega=1 /(2 \varepsilon) .
$$

\footnotetext{
* Received by the editors August 13, 1973.

† Department of Applied Mathematics, Mathematical Centre, Amsterdam, the Netherlands.
} 
Then the function $F$ has to satisfy the following boundary value problem:

$$
\begin{aligned}
& \Delta F(x, y)-\omega^{2} F(x, y)=0, \\
& F(x, 0)=1, \quad F(x, y)=0 \quad \text { if } \phi=\alpha .
\end{aligned}
$$

In general, the solution of an elliptic equation in an unbounded domain is not unique. But, by imposing a condition upon $F$ concerning its growth at infinity, uniqueness can be ensured. We prove the following lemma. The function $I_{0}(\omega r)$ appearing in the lemma is a modified Bessel function satisfying $\Delta u-\omega^{2} u$ $=0, I_{0}(\omega r)>0, I_{0}(\omega r)=\exp (\omega r) / \sqrt{2 \pi \omega r}\left(1+O\left(r^{-1}\right)\right)$ as $r \rightarrow \infty$.

LEMMA. Assume that $u$ is a regular function in $A$ satisfying: (i) $\Delta u-\omega^{2} u=0$, (ii) $u=0$ on the boundary of $A$, (iii) $\lim _{r \rightarrow \infty} u(x, y) / I_{0}(\omega r)=0$. Then $u=0$ in the whole domain $A$.

Proof. Let $v=u / w$, with $w(x, y)=I_{0}(\omega r)$. The function $v$ satisfies the elliptic equation

$$
\Delta v+\frac{2}{w}\left(v_{x} w_{x}+v_{y} w_{y}\right)=0
$$

and $v=0$ on the boundary of $A$. Owing to (iii), for every positive number $\sigma$ we can find $R$ such that $r>R$ implies $|v(x, y)|<\sigma$. Consider the part $\Delta$ of $A$ contained inside a circle with radius $R_{1}>R$ and center at the origin. Then on the boundary of $\Delta$ we have $|v|<\sigma$. According to the maximum principle for elliptic equations in bounded domains, the inequality $|v|<\sigma$ holds in the whole set $\Delta$. For an arbitrary point $\left(x_{0}, y_{0}\right) \in A, R_{1}$ can be chosen large enough for $\Delta$ to contain that point. Then $\left|v\left(x_{0}, y_{0}\right)\right|<\sigma$, and, since $\sigma$ may be arbitrarily small, $v\left(x_{0}, y_{0}\right)=0$ and hence $u\left(x_{0}, y_{0}\right)=0$, which proves the lemma.

A formal solution of the Helmholtz equation in (2.2) may be written as

$$
F(x, y)=\int e^{A z e^{-t}+B \bar{z} e^{t}} f(t) d t,
$$

where $z=x+i y, \bar{z}=x-i y$ and $A, B$ are constants to be specified. It can be easily verified that $F$ in (2.3) satisfies the equation in (2.2) by writing Laplace's operator as

$$
\frac{\partial^{2}}{\partial x^{2}}+\frac{\partial^{2}}{\partial y^{2}}=4 \frac{\partial^{2}}{\partial z \partial \bar{z}} .
$$

Performing the differentiation in (2.3) we obtain $\triangle F=4 A B F$. Hence, if $4 A B=\omega^{2}$, then $F$ satisfies the equation in (2.2). Taking $A=\frac{1}{2} i \omega, B=-\frac{1}{2} i \omega, z=r e^{i \phi}$, we have

$$
F(x, y)=\int e^{-i \omega r \sinh t} f(t+i \phi) d t
$$

By changing (2.3) into

$$
\int e^{A \bar{z} e^{-t}+B z e^{t}} g(t) d t
$$


we obtain a representation as in (2.4), but now with $g(t-i \phi)$ in the integrand. Hence, a formal solution of the Helmholtz equation can be represented by

$$
F(x, y)=\int e^{-i \omega r \sinh t}\{f(t+i \phi)+g(t-i \phi)\} d t .
$$

For suitable choices of $f$ and $g$ the expression $f(t+i \phi)+g(t-i \phi)$ becomes the real (or imaginary) part of a holomorphic function of the complex variable $\zeta=t+i \phi$ (with real $t$ and $\phi$ ). In that case this expression is a harmonic function of the variables $t$ and $\phi$.

To solve the boundary value problem (2.2) we choose a representation of the following kind:

$$
F(x, y)=\int_{-\infty}^{\infty} e^{-i \omega r \sinh t} U(t, \phi) d t,
$$

where $U$ is harmonic (but not necessarily holomorphic) in the strip

$$
B=\{t, \phi \mid-\infty<t<\infty, 0<\phi<\alpha\} .
$$

In view of the boundary conditions of $F$ (see (2.2)) we obtain for $U$ the following boundary value problem:

$$
\begin{aligned}
& \frac{\partial^{2} U}{\partial t^{2}}+\frac{\partial^{2} U}{\partial \phi^{2}}=0 \quad \text { in } B \\
& U(t, 0)=\delta(t), \quad U(t, \alpha)=0 .
\end{aligned}
$$

Solutions of the Laplace equation in the strip $B$ with Dirichlet boundary conditions can be obtained by using the conformal mapping $\eta=\exp (\pi \zeta / \alpha)$, which gives a potential problem in a half-plane. In the underlying case we choose a more direct method.

Suppose $U(t, \phi)=\operatorname{Re} f(\zeta), \zeta=t+i \phi$. Then the singularity of $U$ in $\zeta=0$ may be represented by $(i / \pi)(1 / \zeta)$ and $f$ may be constructed by the principle of reflection.

$$
f(\zeta)=\frac{i}{\pi}\left[\frac{1}{\zeta}+\sum_{k=1}^{\infty} \frac{2 \zeta}{\zeta^{2}+4 k^{2} \alpha^{2}}\right]=\frac{i}{\pi} \frac{\mu}{e^{\mu \zeta}-1}
$$

where

$$
\mu=\pi / \alpha .
$$

The real part of $f$ is then given by

$$
U(t, \phi)=\frac{1}{2 \alpha} \frac{\sin \mu \phi}{\cosh \mu t-\cos \mu \phi} .
$$

Hence

$$
F(x, y)=\frac{\sin \mu \phi}{2 \alpha} \int_{-\infty}^{\infty} e^{-i \omega r \sinh t} \frac{d t}{\cosh \mu t-\cos \mu \phi}
$$


This function is bounded by the expression

$$
\frac{\sin \mu \phi}{2 \alpha} \int_{-\infty}^{\infty} \frac{d t}{\cosh \mu t-\cos \mu \phi}=\frac{\alpha-\phi}{\alpha},
$$

and hence the conditions for uniqueness are fulfilled. With this function $F$ the solution of $(1.1)$ is

$$
\Phi_{\varepsilon}(x, y)=1-\frac{\sin \mu \phi}{2 \alpha} e^{\omega r \sin \phi} \int_{-\infty}^{\infty} e^{-i \omega r \sinh t} \frac{d t}{\cosh \mu t-\cos \mu \phi} .
$$

This representation of the solution of the singular perturbation problem (1.1) will be the starting point of the investigations on the asymptotic behavior of $\Phi_{\varepsilon}(x, y)$ for $\varepsilon \rightarrow 0$ (i.e., $\omega \rightarrow \infty$ ). The integral in (2.8) will be evaluated by saddle point methods. The saddle points of the function $e^{-i \omega r \sinh t}$ are located at the zeros of $\cosh t$, i.e., at $t_{n}=i\left(\frac{1}{2} \pi+n \pi\right), n$ being an integer. The steepest descent lines are lines parallel to the real $t$-axis through $t_{n}$. If convergence is not disturbed, the path of integration of the integral in (2.8) may be shifted towards a steepest descent line. With this condition, only the saddle point at $-\frac{1}{2} i \pi$ can be considered.

By shifting the path of integration of (2.8) downwards to the line $\operatorname{Im} t=-\frac{1}{2} \pi$, singularities of the integrand may be passed. The singularities in this case are poles due to the zeros of

$$
\cosh \mu t-\cos \mu \phi=2 \sin \left(\frac{1}{2} \mu(\phi+i t)\right) \sin \left(\frac{1}{2} \mu(\phi-i t)\right)
$$

The zeros are $t_{k}=-i(\phi+2 \alpha k)$ and $\bar{t}_{k}$ (the complex conjugate of $t_{k}$ ) for integer values of $k$. The following poles are important in our problem:

$$
\begin{array}{ll}
t_{k}=-i(\phi+2 \alpha k) & \text { for } k=0,1,2, \cdots, \\
\bar{t}_{k}=i(\phi+2 \alpha k) & \text { for } k=-1,-2,-3, \cdots .
\end{array}
$$

Only these poles may be located in $\left[0,-\frac{1}{2} i \pi\right]$, the number of which is dependent on $\alpha$. We consider two different cases: $\frac{1}{2} \pi<\alpha<2 \pi$ and $0<\alpha<\frac{1}{2} \pi$. The first case is simpler than the second one, and will be considered first.

3. The case $\frac{1}{2} \pi<\alpha<2 \pi$. For $0<\phi<\alpha$, only the pole $t_{0}=-i \phi$ of (2.10) may be located in the interval $\left[0,-\frac{1}{2} i \pi\right]$. For values of $\phi$ close to $\frac{1}{2} \pi$, the pole $t_{0}$ lies close to the saddle point at $t=-\frac{1}{2} i \pi$. In order to obtain an asymptotic expansion of $\Phi_{\varepsilon}$ which holds uniformly for all values of $\phi$ in $[0, \alpha]$, we use the same method as in [4].

Essential to this method is the regularization of the integrand in (2.8) by an appropriate function. This will be done by determining a constant (i.e., independent of $t) c$ such that the function

$$
\frac{\sin \mu \phi}{2 \alpha} \frac{1}{\cosh \mu t-\cos \mu \phi}-\frac{c}{\sinh \frac{1}{2}(t+i \phi)}
$$

is regular at $t=-i \phi$. By calculating the residues at $t=-i \phi$ of both members 
of (3.1), we infer $c=i / 4 \pi$. The function $\Phi_{\varepsilon}$ of (2.8) can now be written as

$$
\Phi_{\varepsilon}(x, y)=1+\frac{e^{\omega y}}{4 \pi i} \int_{-\infty}^{\infty} e^{-i \omega r \sinh t} \frac{d t}{\sinh \frac{1}{2}(t+i \phi)}-e^{\omega y} \int_{-\infty}^{\infty} e^{-i \omega r \sinh t} g(t) d t
$$

where

$$
g(t)=U(t, \phi)+\frac{1}{4 \pi i \sinh \frac{1}{2}(t+i \phi)},
$$

and $U$ is defined in (2.7).

The first integral in (3.2) can be evaluated by means of the following formula:

$$
\begin{gathered}
F(r, \gamma)=\frac{1}{2 \pi i} \int_{-\infty}^{\infty} e^{-r \cosh u} \frac{d u}{\sinh \frac{1}{2}(u-i \gamma)}=e^{-r \cos \gamma} \operatorname{erfc}\left(\sqrt{2 r} \sin \frac{1}{2} \gamma\right), \\
0<\gamma<2 \pi
\end{gathered}
$$

where

$$
\operatorname{erfc}(z)=\frac{2}{\sqrt{\pi}} \int_{z}^{\infty} e^{-t^{2}} d t
$$

Formula (3.4) can be found in Lauwerier's papers [3] and is also used in [4]. A proof of (3.4) is easily obtained by verifying that

$$
\frac{\partial}{\partial r}\left\{e^{r \cos \gamma} F(r, \gamma)\right\}=-2 \sqrt{\frac{2 \pi}{r}} \sin \frac{1}{2} \gamma e^{-r(1-\cos ;)} .
$$

Now, letting $u=t+\frac{1}{2} i \pi, \gamma=5 \pi / 2-\phi$ and using

$$
\operatorname{erfc}(-z)=2-\operatorname{erfc}(z)
$$

we obtain

$$
1+\frac{e^{\omega r \sin \phi}}{4 \pi i} \int_{-\infty}^{\infty} e^{-i \omega r \sinh t} \frac{d t}{\sinh \frac{1}{2}(t+i \phi)}=\frac{1}{2} \operatorname{erfc}(z)
$$

where

$$
z=\sqrt{2 \omega r} \sin \frac{1}{2}\left(\frac{1}{2} \pi-\phi\right) .
$$

Formula (3.6) holds for $\frac{1}{2} \pi<\phi<2 \pi$. But by considering complex values of $\phi$ and by using analytic continuation, (3.6) can be shown to hold for $0<\operatorname{Re} \phi<2 \pi$.

The function $g$ of (3.3) is regular for $t \in\left[0,-\frac{1}{2} i \pi\right]$ and $0<\phi<\alpha\left(\frac{1}{2} \pi<\alpha<2 \pi\right)$. Hence, by shifting the path of integration in the second integral of (3.2) downwards to the line $\operatorname{Im} t=-\frac{1}{2} \pi$, we obtain

$$
\Phi_{\varepsilon}(x, y)=\frac{1}{2} \operatorname{erfc}(z)-e^{\omega y} \int_{-\infty}^{\infty} e^{-\omega r \cosh t} g\left(t-\frac{1}{2} i \pi\right) d t .
$$

So far, large values of $\omega$ (i.e., small values of $\varepsilon$ ) have not been considered. The representation (3.8) of the solution of the boundary value problem is the exact representation. In order to get an asymptotic expansion of $\Phi_{\varepsilon}$, we expand $g$ 
into a series

$$
g\left(t-\frac{1}{2} i \pi\right)=\cosh \frac{1}{2} t \sum_{k=0}^{\infty} c_{k}\left(\sinh \frac{1}{2} t\right)^{k} .
$$

Substitution of this series in (3.8) and interchanging the order of summation and integration yields

$$
\Phi_{\varepsilon}(x, y) \simeq \frac{1}{2} \operatorname{erfc}(z)-2 e^{-\omega r(1-\sin \phi)} \sum_{k=0}^{\infty} c_{2 k} \frac{\Gamma\left(k+\frac{1}{2}\right)}{(2 \omega r)^{k+1 / 2}}
$$

as $\omega r \rightarrow \infty$, uniformly with respect to $\phi, 0 \leqq \phi \leqq \alpha$.

The expansion in (3.10) breaks down if $\alpha \rightarrow \frac{1}{2} \pi$. Namely, the function $g\left(t-\frac{1}{2} i \pi\right)$ has a pole at $i\left(\phi+\frac{1}{2} \pi-2 \alpha\right)$. For $\phi=\alpha$, this pole is located at $i\left(\frac{1}{2} \pi-\alpha\right)$ and if $\alpha \rightarrow \frac{1}{2} \pi$ this pole approaches the origin. As a consequence, the coefficients $c_{k}$ in (3.9) and (3.10) tend to infinity if $\alpha \rightarrow \frac{1}{2} \pi$. For this question the reader is referred to $\S 5$.

The most important term in the asymptotic expansion (3.10) is

$$
\Phi_{\varepsilon}^{(0)}(x, y) \equiv \frac{1}{2} \operatorname{erfc}(z)
$$

with $z$ defined in (3.7). This term exhibits the behavior of $\Phi_{\varepsilon}$ in the neighborhood of $\phi=\frac{1}{2} \pi$, i.e., along the $y$-axis in the $x, y$-plane. Just as in the quarter-plane case, this term leads to a parabolic boundary layer, situated along the positive $y$-axis. In this domain, for large values of $\omega r$, the function $\Phi_{\varepsilon}^{(0)}$ (and hence $\Phi_{\varepsilon}$ ) rapidly changes from the value $\frac{1}{2}$ to very small values $(x>0)$ or to values close to 1 $(x<0)$. This boundary layer is called a "free" or "internal" boundary layer, since it is not located along the boundary of the domain $A$ for which the boundary value problem (1.1) is defined.

Along the boundary $\phi=\alpha$ boundary layers do not occur, as can be seen from (3.10). Namely, if $\phi \simeq \alpha\left(\alpha>\frac{1}{2} \pi\right)$,

$$
\Phi_{\varepsilon}(x, y)-\Phi_{\varepsilon}^{(0)}(x, y)=O\left((\omega r)^{-N}\right)
$$

as $\omega r \rightarrow \infty$, for any positive $N$ and all $\phi, \frac{1}{2} \pi+\delta \leqq \phi \leqq \alpha$, where $\delta$ is a small positive constant. For these values of $\phi$ we also have

$$
\Phi_{\varepsilon}^{(0)}(x, y)-1=O\left((\omega r)^{-N}\right)
$$

as follows from

$$
\operatorname{erfc}(-z)=2-\operatorname{erfc}(z)
$$

and from the well-known asymptotic formula

$$
\operatorname{erfc}(z)=\frac{1}{\sqrt{\pi} z} e^{-z^{2}}\left(1+O\left(z^{-2}\right)\right)
$$

as $z \rightarrow+\infty$.

4. The acute angle. In this section we consider values of $\phi$ and $\alpha$ in the range

$$
0 \leqq \phi \leqq \alpha<\frac{1}{2} \pi .
$$


First we determine the number of poles (2.14) located on the imaginary $t$-axis between 0 and $-\frac{1}{2} i \pi$.

We introduce

$$
\lambda \equiv \frac{2 \alpha}{\pi}\left(=\frac{2}{\mu}\right),
$$

so that $0<\lambda<1$. Consequently, we can choose an integer $n \geqq 2$, satisfying

$$
n-1<1 / \lambda \leqq n
$$

We distinguish two cases:

(a) If in (4.3) $n$ is odd, then we have with $k_{0}=\frac{1}{2}(n-1)$,

$$
2 \alpha k_{0}<\frac{1}{2} \pi \leqq\left(2 k_{0}+1\right) \alpha .
$$

Therefore, the pole

$$
t_{k_{0}}=-i\left(\phi+2 \alpha k_{0}\right)
$$

passes through $-\frac{1}{2} i \pi$ when $\phi$ changes from 0 to $\alpha$. If $\phi+2 \alpha k_{0}<\frac{1}{2} \pi$, then $t_{k_{0}} \in\left[0,-\frac{1}{2} i \pi\right]$; if $\phi+2 \alpha k_{0}>\frac{1}{2} \pi$, then $t_{k_{0}} \notin\left[0,-\frac{1}{2} i \pi\right]$. For all values of $\phi$ $(0 \leqq \phi \leqq \alpha)$, we have

$$
t_{k}, \bar{t}_{l} \in\left[0,-\frac{1}{2} i \pi\right] \text { for } k=0,1, \cdots, k_{0}-1, \quad l=1,2, \cdots, k_{0} .
$$

(b) If in (4.3) $n$ is even, we have with $l_{0}=\frac{1}{2} n$,

$$
\left(2 l_{0}-1\right) \alpha<\frac{1}{2} \pi \leqq 2 \alpha l_{0} \text {. }
$$

Therefore, the pole

$$
\bar{t}_{-l_{0}}=i\left(\phi-2 \alpha l_{0}\right)
$$

passes the point $-\frac{1}{2} i \pi$ if $\phi$ changes from 0 to $\alpha$. If $\phi+\frac{1}{2} \pi>2 \alpha l_{0}$, then $\bar{t}_{-l_{0}}$ $\in\left[0,-\frac{1}{2} i \pi\right]$; if $\phi+\frac{1}{2} \pi<2 \alpha l_{0}$, then $\bar{t}_{-l_{0}} \notin\left[0,-\frac{1}{2} i \pi\right]$. For all values of $\phi(0 \leqq \phi \leqq \alpha)$,

$$
t_{k}, \bar{t}_{-l} \in\left[0,-\frac{1}{2} i \pi\right] \text { for } k=0,1, \cdots, l_{0}-1, \quad l=1,2, \cdots, l_{0}-1 .
$$

As in $\S 3$, the poles $t_{k_{0}}$ and $\bar{t}_{-l_{0}}((4.5)$ and (4.7) respectively) can be split off. In this way error functions are introduced. Afterwards the path of integration will be shifted downwards to the line $\operatorname{Im} t=-\frac{1}{2} \pi$. The residues of the poles being passed turn out to be exponential functions. A simple calculation gives the following results. (We distinguish again the two cases (a) and (b).)

$$
\begin{aligned}
\Phi_{\varepsilon}(x, y) & =\sum_{k=1}^{k_{0}} e^{-\omega r(\sin (2 k \alpha-\phi)-\sin \phi\}}-\sum_{k=1}^{k_{0}-1} e^{-\omega r\{\sin (2 k \alpha+\phi)-\sin \phi\}} \\
- & \frac{1}{2} \operatorname{erfc}(z) e^{-\omega r\left(\sin \left(2 \alpha k_{0}+\phi\right)-\sin \phi\right\}}-e^{\omega r \sin \phi} \int_{-\infty}^{\infty} e^{-\omega r \cosh t} g(t) d t
\end{aligned}
$$

where $k_{0}$ is specified in (4.4), $z=\sqrt{2 \omega r} \sin \frac{1}{2} \gamma$,

$$
g(t)=U\left(t-\frac{1}{2} i \pi, \phi\right)+\frac{1}{4 \pi i \sinh \frac{1}{2}(t-i \gamma)},
$$

and $\gamma=\frac{1}{2} \pi-\phi-2 \alpha k_{0}$. 
(b)

$$
\begin{aligned}
\Phi_{\varepsilon}(x, y) & =\sum_{k=1}^{l_{0}-1} e^{-\omega r\{\sin (2 \alpha k-\phi)-\sin \phi\}}-\sum_{k=1}^{l_{0}-1} e^{-\omega r\{\sin (2 \alpha k+\phi)-\sin \phi\}} \\
+ & \frac{1}{2} \operatorname{erfc}(z) e^{-\omega r\left\{\sin \left(2 \alpha l_{0}-\phi\right)-\sin \phi\right\}}-e^{\omega r \sin \phi} \int_{-\infty}^{\infty} e^{-\omega r \cosh t} g(t) d t,
\end{aligned}
$$

where $l_{0}$ is specified in (4.6), $z=\sqrt{2 \omega r} \sin \frac{1}{2} \gamma$,

$$
g(t)=U\left(t-\frac{1}{2} i \pi, \phi\right)-\frac{1}{4 \pi i \sinh \frac{1}{2}(t-i \gamma)}
$$

and $\gamma=\frac{1}{2} \pi+\phi-2 \alpha l_{0}$.

The representation (4.8) (resp. (4.10)) of $\Phi_{\varepsilon}(x, y)$ is the exact solution. In order to get an asymptotic expansion of $\Phi_{\varepsilon}$ for small values of $\varepsilon$, the function $g$ in (4.9) (resp. (4.11)) may be expanded in the same way as was done for $g$ in (3.9). The asymptotic expansion obtained by interchanging the order of summation and integration (cf. (3.10)) is uniformly valid in $0 \leqq \phi \leqq \alpha$. Just as in the foregoing section, if $\alpha \rightarrow \frac{1}{2} \pi$, the expansion must be reconsidered (see $\S 5$ ).

We conclude this section with some remarks concerning the boundary layer. If $\phi \simeq \alpha$, the asymptotic behavior of $\Phi_{\varepsilon}$ is determined by the first term of the first finite series in (4.8) (resp. (4.10)); the other terms are of lower order. Hence

$$
\Phi_{\varepsilon}(x, y) \simeq e^{-\omega r\{\sin (2 \alpha-\phi)-\sin \phi\}}
$$

as $\omega r \rightarrow \infty, \phi \rightarrow \alpha, \phi \leqq \alpha$. If $\phi<\alpha$, the right-hand side of (4.12) is very small, explicitly

$$
\Phi_{\varepsilon}(x, y)=O\left((\omega r)^{-N}\right)
$$

where $N$ is an arbitrary positive number. This estimate, however, is not uniformly valid in $\phi$. If $\phi \rightarrow \alpha$, the exponential function in (4.12) may not be small at all. We can determine the locus in the $x, y$-plane on which the argument of the exponential function in (4.12) is constant. We infer from

$$
-\omega r\{\sin (2 \alpha-\phi)-\sin \phi\}=-c \quad(c>0)
$$

that the locus is a straight line

$$
y=x \tan \alpha-c / \omega,
$$

which is parallel to the boundary $y=x \tan \alpha$ of the sector $A$. From these aspects we conclude that along the line $y=x \tan \alpha$ a boundary layer of thickness $O(\varepsilon)$ is located.

The term with the error function in (4.8) (resp. 4.10)) is asymptotically of lower order than the term in (4.12). The error function part, however, is of great importance. The error function changes rapidly at $\phi=\frac{1}{2} \pi-2 \alpha k_{0}$ (resp. $2 \alpha l_{0}-\frac{1}{2} \pi$ ), but the effect is damped by the exponential function contained in this term. This term is gaining in influence if $\alpha \rightarrow \frac{1}{2} \pi$ and the (hidden) internal boundary layer due to the error function comes to light if $\alpha \rightarrow \frac{1}{2} \pi$ (see $\S 5$ ).

5. The almost right angle. In $\S \S 3$ and 4 we discussed the asymptotic behavior of $\Phi_{\varepsilon}(\varepsilon \rightarrow 0)$ for values of $\alpha$ larger, respectively smaller than $\frac{1}{2} \pi$. However, the 
expansion in (3.9) and the expansions which can be derived from (4.8) (resp. (4.10)) in an analogous way, are not valid if $\phi, \alpha \rightarrow \frac{1}{2} \pi$. since the function $g(t)$ has a singularity, which tends to zero for $\phi, \alpha \rightarrow \frac{1}{2} \pi$. In this section we shall give the asymptotic representation of $\Phi_{\varepsilon}$ holding for all $\alpha \in\left[\frac{1}{2} \pi-\delta, \frac{1}{2} \pi+\delta\right]$, where $0<\delta<\frac{1}{4} \pi$.

Suppose first that $\frac{1}{4} \pi<\alpha<\frac{1}{2} \pi$. In this case the results of $\S 4$ can be used. In (4.2) we have $\frac{1}{2}<\lambda<1$ and in (4.3) we have $n=2$. Hence, the (b)-case applies and from (4.6) it follows that $l_{0}=1$. Thus (4.8) becomes

$$
\Phi_{\varepsilon}(x, y)=\frac{1}{2} \operatorname{erfc}(z) e^{-\omega r\{\sin (2 x-\phi)-\sin \phi\}}+e^{(\omega) \sin \phi} \int_{-\infty}^{\infty} e^{-(\omega) \cosh t} g(t) d t,
$$

where $z=\sqrt{2 \omega r} \sin \frac{1}{2} \gamma, \gamma=\phi+\frac{1}{2} \pi-2 \alpha$ and $g$ is defined in (4.9). The function $g$ has a pole in $i\left(\frac{1}{2} \pi-\phi\right)$, corresponding to $t_{k}$ in (2.14) with $k=0$. In (4.8) this pole has no influence since $\alpha$ is constant. If, alternatively, $\alpha \simeq \frac{1}{2} \pi$, this singularity is close to the origin for values of $\phi$ close to $\alpha$. This pole can be split off and so another error function is introduced.

Suppose next $\frac{1}{2} \pi<\alpha<2 \pi$. The function $g$ in (3.3) has a pole in $i\left(\phi+\frac{1}{2} \pi-2 \alpha\right)$, corresponding to $\bar{t}_{k}$ in (2.14) with $k=-1$. Again, for $\alpha \simeq \frac{1}{2} \pi$, this pole is close to the origin for values of $\phi$ close to $\alpha$.

Combining the two cases, we have

$$
\begin{aligned}
\Phi_{\varepsilon}(x, y)= & \frac{1}{2} \operatorname{erfc}(\zeta)+\frac{1}{2} \operatorname{erfc}(z) e^{-\omega r\{\sin (2 \alpha-\phi)-\sin \phi\}} \\
& -e^{\omega r \sin \phi} \int_{-\infty}^{\infty} e^{-\omega r \cosh t} h(t) d t,
\end{aligned}
$$

where

$$
\begin{gathered}
h(t)=U\left(t-\frac{1}{2} i \pi, \phi\right)-\frac{1}{4 \pi i \sinh \frac{1}{2}(t-i \gamma)}+\frac{1}{4 \pi i \sinh \frac{1}{2}\left(t-i\left(\frac{1}{2} \pi-\phi\right)\right)}, \\
\gamma=\phi+\frac{1}{2} \pi-2 \alpha, \quad z=\sqrt{2 \omega r} \sinh \frac{1}{2} \gamma, \quad \zeta=\sqrt{2 \omega r} \sin \frac{1}{2}\left(\frac{1}{2} \pi-\phi\right) .
\end{gathered}
$$

The asymptotic expansion of $\Phi_{\varepsilon}$ for large $\omega r$ may be derived by expanding $h$ in the same way as was done for $g$ in (3.8). The asymptotic expansion so obtained holds uniformly in $0 \leqq \phi \leqq \alpha, \frac{1}{2} \pi-\delta \leqq \alpha \leqq \frac{1}{2} \pi+\delta$, where $0<\delta<\frac{1}{4} \pi$. If $\alpha=\frac{1}{2} \pi$, (the quarter-plane, see [4]) we have $\gamma=\phi-\frac{1}{2} \pi$ and

$$
\Phi_{\varepsilon}(x, y)=\operatorname{erfc}\left(\sqrt{2 \omega r} \sin \frac{1}{2}\left(\frac{1}{2} \pi-\phi\right)\right)-e^{\omega r \sin \phi} \int_{-\infty}^{\infty} e^{-\omega r \cosh t} h(t) d t,
$$

where the integral equals the corresponding integral of reference [4] (formula (4.6)).

The significant terms of (5.2) are the two terms with the error functions. For $\alpha<\frac{1}{2} \pi$, the second term may be connected with the "linear" boundary layer along $\phi=\alpha$ and the (hidden) internal layer at $\phi=2 \alpha-\frac{1}{2} \pi$. The first one may be connected with an external parabolic boundary layer outside the sector $A$. This boundary layer has no influence since it is situated outside the domain of definition. If however $\alpha \rightarrow \frac{1}{2} \pi\left(\alpha<\frac{1}{2} \pi\right)$ this boundary layer enters the domain $A$, and coalesces (in the limit $\alpha=\frac{1}{2} \pi$ ) with both the "linear" boundary layer and the 
(hidden) internal layer at $\phi=2 \alpha-\frac{1}{2} \pi$ (see Fig. 1).

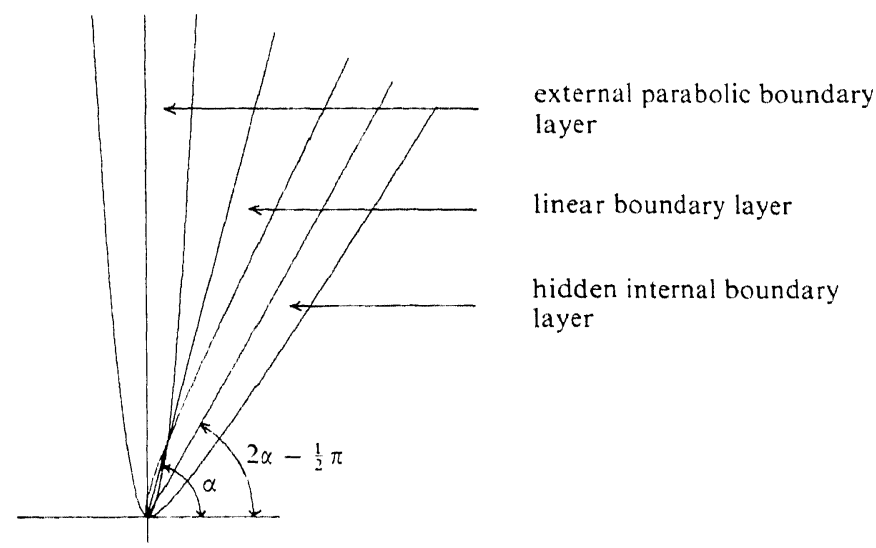

FIG. 1. $\alpha<\frac{1}{2} \pi$

For $\alpha>\frac{1}{2} \pi$, the first term in (5.2) may be associated with the internal parabolic boundary layer inside the sector at $\phi=\frac{1}{2} \pi$. The second one may be connected with a boundary layer outside $A$, which is situated at $\phi=2 \alpha-\frac{1}{2} \pi$ and which enters the domain if $\alpha \rightarrow \frac{1}{2} \pi$. In the limit $\left(\alpha=\frac{1}{2} \pi\right)$ the two types of boundary layers pass into the parabolic boundary layer along $\phi=\alpha=\frac{1}{2} \pi$ (see Fig. 2).

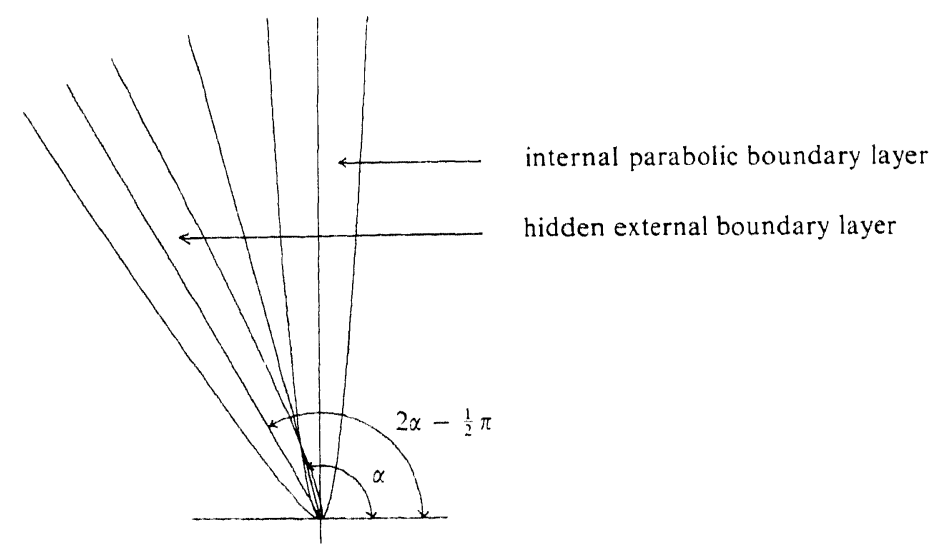

FIG. 2. $\alpha>\frac{1}{2} \pi$

From our remarks on the coincidence of the boundary layers it may be established that the parabolic boundary layer of the quarter-plane is a particular case of parabolic boundary layers for the almost right angle. For other aspects, the reader is referred to Grasman [2], where the case of almost characteristic boundaries is treated with coordinate-stretching techniques. 
6. An analogous problem. An analogous, but much simpler, problem is encountered in looking for the asymptotic expansion of the solutions of the boundary value problem

$$
\varepsilon \Delta \Phi_{\varepsilon}(x, y)-\mu \frac{\partial}{\partial x} \Phi_{\varepsilon}(x, y)-\lambda \frac{\partial}{\partial y} \Phi_{\varepsilon}(x, y)=0
$$

in the quarter-plane $A=\{x, y \mid x \geqq 0, y \geqq 0\}$ with boundary conditions

$$
\Phi_{\varepsilon}(0, y)=1, \quad \Phi_{\varepsilon}(x, 0)=0 .
$$

In (6.1), $\mu$ and $\lambda$ are numbers independent of $x$ and $y$. The characteristics of the reduced equation $(\varepsilon=0$ in $(6.1))$

$$
\mu \frac{\partial \phi}{\partial x}+\lambda \frac{\partial \phi}{\partial y}=0
$$

are the lines $y=(\lambda / \mu) x+c$. For small values of $\mu$, the characteristics of (6.2) are nearly parallel to the boundary line $x=0$. Therefore, for small values of $\mu$ it is expected that again two error functions appear in the asymptotic expansion of $\Phi_{\varepsilon}$ (for $\varepsilon \rightarrow 0$ ). As can be verified by the methods of $\S 2$, the function $\Phi_{\varepsilon}$ can be written down as follows:

$$
\Phi_{\varepsilon}(x, y)=e^{\omega r \sin (\phi+\beta)} \int_{-\infty}^{\infty} e^{-i \omega r \sinh t} U(t, \phi) d t,
$$

where

$$
\begin{aligned}
x=r \cos \phi, & y=r \sin \phi, \quad \lambda=\rho \cos \beta, \quad \mu=\rho \sin \beta, \quad \omega=\rho /(2 \varepsilon), \\
U(t, \phi) & =\frac{1}{\pi} \operatorname{Re}\left\{\tan \frac{1}{2}(i t+\phi+\beta)+\tan \frac{1}{2}(i t+\phi-\beta)\right\} \\
& =\frac{1}{\pi} \frac{\sin (\phi+\beta)}{\cosh t+\cos (\phi+\beta)}+\frac{1}{\pi} \frac{\sin (\phi-\beta)}{\cosh t+\cos (\phi-\beta)} .
\end{aligned}
$$

7. Concluding remarks. In this paper we used analytical methods which only can be applied on singular perturbation problems with simple differential operators, boundary values and suitable domains of definition. The methods cannot easily be generalized for other problems. In treating the relatively simple problems, however, we have a different aim.

For instance, our approach of the problem gives results which are not easily noticed by using the usual singular perturbation techniques. We allude to the existence of the hidden boundary layer along the line $\phi=2 \alpha-\frac{1}{2} \pi$ (see Fig. 1 and the conclusion of $\S 4$ ). This aspect is not discussed in boundary layer techniques, since the function $\Phi_{\varepsilon}$ is asymptotically of order zero in the neighborhood of this internal layer. In order to obtain a uniform asymptotic expansion with respect to $\phi$ (in $0 \leqq \phi \leqq \alpha<\frac{1}{2} \pi$ ), the error function corresponding with this layer cannot be omitted.

Further we shall point to the case of an almost characteristic boundary (see $\S 5$ ). In a clear and simple way the asymptotic behavior of $\Phi_{\varepsilon}$ can be described, 
using our methods. Also the way in which the various boundary layers pass into each other is apparent.

An important disadvantage of our methods is the following. The asymptotic expansions are derived for large values of $\omega r$. Hence, the results of our paper do not hold in an $\varepsilon$-neighborhood of the origin. This domain is very small but it is very interesting, since in this part of the $x, y$-plane the boundary layers arise. It is possible to give expansions which represent the behavior of $\Phi_{\varepsilon}$ close to the origin, but it seems better to us to tackle this problem with coordinate stretching techniques. This aspect, however, falls outside the scope of this paper.

Our results can successfully be applied in general singular perturbation problems, which yield reduced problems with relatively simple differential operators, boundary values and domains of definition. With these reduced problems the local behavior of the solutions are investigated.

Acknowledgment. The author wishes to express his gratitude to Professor H. A. Lauwerier for suggesting this investigation and for helpful discussions.

\section{REFERENCES}

[1] D. VAN DANTZIG, Solution of the equation of Helmholtz in an angle with vanishing directional derivatives along each side, Nederl. Akad. Wetensch. Proc. Ser. A., 61 (1958), pp. 384-398.

[2] J. Grasman, An elliptic singular perturbation problem with almost characteristic boundaries, Rep. TN 67/72, Math. Centre, Amsterdam, 1972; J. Math. Anal. Appl., 46 (1974), to appear.

[3] H. A. LAUWERIER, Solutions of the equation of Helmholtz in an angle. $I, I I, I I I, I V, V, V I$, Nederl. Akad. Wetensch. Proc. Ser. A, 62 (1959), pp. 475-488; 63 (1960), pp. 355-372; 64 (1961), pp. 123-140, 348-359; 65 (1962), pp. 93-99, 473-483.

[4] N. M. Tемме, Analytical methods for a singular perturbation problem. The quarter-plane, Rep. TW 125/71, Math. Centre, Amsterdam, 1971. 